\title{
Image-Guided Interventions in Oncology: A Look into the Future?
}

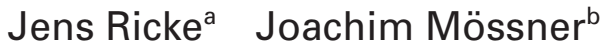 \\ ${ }^{a}$ Department of Radiology and Nuclear Medicine, University Clinic Magdeburg, Magdeburg, Germany \\ ${ }^{\mathrm{b}}$ Division of Gastroenterology and Rheumatology, Department of Internal Medicine, Neurology and Dermatology, University Hospital of Leipzig, \\ Leipzig, Germany
}

For many years, the diagnosis of metastasis in gastrointestinal tumours was deemed fatal for the patient. Nearly 40 years ago, there was an increasing number of reports according to which patients were surviving in the long term or healed after resection of solitary, small liver metastases. The accounts of experienced surgeons who actively worked during those days suggest the incredulity with which such reports were perceived by the oncology community. Since then, not only the readiness to perform an increasingly pervasive tumour resection of liver metastases but also the specific surgical technique for this has developed enormously. In the next step, it was mainly lung metastasis that could be removed, enabling to promise the patient an improved prognosis if the tumour biology was right. In parallel to this and dating back to the 1990s, locally ablative image-guided procedures such as radiofrequency ablation developed, minimizing the access and ablation trauma and permitting a lower strain for the patient. This made it possible to use such procedures even in patients with severe comorbidities.

A lot of time has passed since then. In addition to the perpetually progressing development of increasingly better imaging by means of computed tomography (CT), magnetic resonance imaging (MRI), or high-resolution ultrasound, as well as the introduction of increasingly perfect methods of celldestroying energy application (e.g. microwave, stereotactic radiation, image-guided brachytherapy), particularly new oncological concepts are becoming more and more interesting. The concept of 'deepness of response', where quick, intense reaction with clear tumour reduction under systemic therapy has a beneficial influence on survival, is of special importance for local ablation techniques. Why should the deepness of response, as it has been documented in detail for colorectal carcinoma in different studies, not also be valid for other solid tumours? Why would this deepness of response not be achievable much faster and more reliably by massive tumour-ablative measures than by systematic chemotherapy? Another phenomenon not yet understood in its complexity is that of 'oligometastasis'. It is unclear at this time which biological markers or indicators describe patients profiting from locally or regionally ablative measures in spite of multiple metastases or various organ systems being affected.

This issue of Viszeralmedizin does not try to give any definitive answers to the questions asked in this editorial - this is impossible at the current state of science. The contributions, and specifically the interdisciplinary discussion, however, clearly present the questions that we need to answer now and in the near future in order to achieve the next, truly great advance in improving survival in metastasis-forming gastrointestinal tumours. The questions that you will find in the articles together with some initial answers range from the ideal technique for microtherapeutic tumour destruction to the best indication to a system of suitable study endpoints in order to actually document which patients will truly benefit from such novel therapy options - to be viewed only holistically in combination with systemic measures.

\section{KARGER \\ Fax +497614520714 \\ Information@Karger.com}

www.karger.com
(C) 2014 S. Karger GmbH, Freiburs

1662-6664/14/0304-0230\$39.50/0

Accessible online at:

www.karger.com/vim
Prof. Dr. med. Jens Ricke

Klinik für Radiologie und Nuklearmedizin

Universitätsklinikum Magdeburg AöR

Leipziger Straße 44, 39120 Magdeburg, Deutschland

jens.ricke@med.ovgu.de 